\title{
On the constructive investigation of a class of linear boundary value problems for $n$th order differential equations with deviating arguments
}

\section{Aleksandr Rumyantsev}

\section{"Correspondence:}

arumyanzev@gmail.com Department of Applied

Mathematics and Informatics, Perm State University, Bukireva street, 15 , Perm, 614990, Russia

\begin{abstract}
A constructive technique for the study of boundary value problems for $n$th order differential equations with deviating arguments is described. A computer-assisted proof of the correct solvability of the considered problem is given.

MSC: Primary 34B05; 34K06; 65L10; secondary 34K10; 65L70

Keywords: differential equations with deviating arguments; boundary value problems; constructive methods; unique solvability; approximate solution
\end{abstract}

\section{Introduction}

Let us cite some facts from the theory of functional differential equations [1] and the general description of the constructive approach to the investigation of boundary value problems for such equations $[2,3]$.

Consider the linear boundary value problem

$$
\mathcal{L} y=f, \quad \ell y=\beta,
$$

where $\mathcal{L}: D S_{p}^{n}[0, T](m) \rightarrow L_{p}^{n}[0, T]$ is a bounded linear operator, $\ell: D S_{p}^{n}[0, T](m) \rightarrow R^{n}$ is a bounded linear vector functional, and $f \in L_{p}^{n}[0, T], \beta \in R^{m n+n} . R^{n}$ denotes the linear space of real columns $\alpha$, where $\alpha=\operatorname{col}\left\{\alpha_{1}, \ldots, \alpha_{n}\right\}$ with the norm $\|\alpha\|_{n}=\max _{1 \leq i \leq n}\left|\alpha_{i}\right| ;\lfloor\alpha\rfloor \stackrel{\text { def }}{=}$ $\operatorname{col}\left\{\left|\alpha_{1}\right|, \ldots,\left|\alpha_{n}\right|\right\} ; L_{p}^{n}[0, T], 1 \leq p<\infty$, denotes the Banach space of measurable functions $z:[0, T] \rightarrow R^{n}, z(\cdot)=\operatorname{col}\left\{z_{1}(\cdot), \ldots, z_{n}(\cdot)\right\}$, such that

$$
\|z\|_{L_{p}^{n}[0, T]}=\max _{1 \leq i \leq n}\left(\int_{0}^{T}\left|z_{i}(s)\right|^{p} d s\right)^{\frac{1}{p}}<+\infty
$$

let us fix a collection of points $0=t_{0}<t_{1}<\ldots<t_{m}<t_{m+1}=T$. Put $B_{q}=\left[t_{q-1}, t_{q}\right), q=1, \ldots, m$; $B_{m+1}=\left[t_{m}, T\right]$

$$
\chi_{q}(t)= \begin{cases}1, & \text { if } t \in B_{q}, \\ 0, & \text { if } t \notin B_{q} ;\end{cases}
$$

O2014 Rumyantsev; licensee Springer. This is an Open Access article distributed under the terms of the Creative Commons Attribution License (http://creativecommons.org/licenses/by/2.0), which permits unrestricted use, distribution, and reproduction in any medium, provided the original work is properly cited. 
$D S_{p}^{n}[0, T](m)$ denotes the Banach space of all functions $y:[0, T] \rightarrow R^{n}$ with $\dot{y} \in L_{p}^{n}[0, T]$ and the representation:

$$
y(t)=y(0)+\int_{0}^{t} \dot{y}(s) d s+\sum_{q=1}^{m} \chi_{\left[t_{q}, T\right]}(t) \Delta y\left(t_{q}\right)
$$

where $\Delta y\left(t_{q}\right)=y\left(t_{q}\right)-y\left(t_{q}-0\right) ; \chi_{\left[t_{q}, T\right]}(t)=\left\{\begin{array}{ll}1, & \text { if } t \in\left[t_{q}, T\right], \\ 0, & \text { if } t \notin\left[t_{q}, T\right] ;\end{array} \stackrel{\text { def }}{=} \operatorname{col}\left\{y(0), \Delta y\left(t_{1}\right), \ldots, \Delta y\left(t_{m}\right)\right\}\right.$, with the norm

$$
\|y\|_{D S_{p}^{n}[0, T]}(m)=\|\dot{y}\|_{L_{p}^{n}[0, T]}+\|\Delta y\|_{m n+n} .
$$

We assume that the principal boundary value problem

$$
\mathcal{L} y=f, \quad \Delta y=\alpha, \quad f \in L_{p}^{n}[0, T], \alpha \in R^{m n+n},
$$

is correctly solvable. Then under these assumptions there exists an $(m n+n) \times(m n+n)$ fundamental matrix $\mathbf{Y}$ for the homogeneous equation:

$$
\mathcal{L} y=0 .
$$

The following statement holds: the problem (1) is uniquely solvable for any $f, \alpha$ if and only if the $(m n+n) \times(m n+n)$ matrix $\Gamma$,

$$
\Gamma \stackrel{\text { def }}{=} \ell \mathbf{Y}
$$

(each column of the $(m n+n) \times(m n+n)$ matrix $\boldsymbol{\Gamma}$ is the result of applying of the functional $\ell$ to the corresponding column of the matrix $\mathbf{Y}$ ) is invertible. The problem (2) is correctly solvable for a broad class of equations, including

- the ordinary differential equation

$$
\begin{aligned}
& \mathcal{L} y \stackrel{\text { def }}{=} \dot{y}(t)+P(t) y(t)=f(t), \quad t \in[0, T] ; \\
& P(\cdot)=\left\{p(\cdot)_{i j}\right\}_{i, j=1}^{n}, \quad p_{i j} \in L_{p}^{1}[0, T] ;
\end{aligned}
$$

- and a differential equation with concentrated delays

$$
\begin{aligned}
& \mathcal{L} y \stackrel{\text { def }}{=} \dot{y}_{i}(t)+\sum_{j=1}^{n} p_{i j}(t) y_{j}\left[h_{i j}(t)\right]=f_{i}(t), \quad t \in[0, T] ; \\
& h_{i j}(\xi)=0, \quad \xi<0, \\
& p_{i j} \in L_{p}^{1}[0, T], \quad h_{i j} \leq t, \quad i, j=1, \ldots n .
\end{aligned}
$$

It should be noted that in the more general case of a differential equation with deviating arguments, the problem (2) does not have this property and some further investigation is required. For example, define the space $D S_{1}^{1}(1)$ on the partition $\left[0, \frac{1}{2}, 1\right]$ and consider the principal boundary value problem

$$
\dot{x}(t)=x(1)+f(t), \quad t \in[0,1], \quad \Delta x=\operatorname{col}\{\alpha, 0\}, \quad f \in L_{1}^{1}[0,1], \alpha \in R .
$$

It easy to see that this problem has a unique solution only for $\alpha=-\int_{0}^{1} f(s) d s$. 
The key idea of the constructive study of the solvability of the problem (1) is as follows.

- Two $(m n+n) \times(m n+n)$ matrices, ${ }^{a} \boldsymbol{\Gamma}$ and ${ }^{v} \boldsymbol{\Gamma}$, with rational elements are constructed according to a specially developed procedure based on a computer-assisted proof, such that

$$
\left\lfloor\boldsymbol{\Gamma}-{ }^{a} \boldsymbol{\Gamma}\right\rfloor \leq\left\lfloor{ }^{v} \boldsymbol{\Gamma}\right\rfloor
$$

let $R^{n \times n}$ denotes the linear space of real $n \times n$-matrices $\mathbf{A}=\left\{a_{i j}\right\}_{i, j=1}^{n}$ with the norm

$$
\|\mathbf{A}\|_{R^{n \times n}}=\max _{1 \leq i \leq n} \sum_{j=1}^{n}\left|a_{i j}\right| ;\lfloor\mathbf{A}\rfloor \stackrel{\text { def }}{=}\left\{\left|a_{i j}\right|\right\}_{i, j=1}^{n} \text {. }
$$

- The invertibility of the matrix ${ }^{a} \boldsymbol{\Gamma}$ is verified using exact arithmetic.

- If there exists an inverse matrix ${ }^{a} \boldsymbol{\Gamma}^{-1}$, it should be checked whether

$$
\|v \boldsymbol{\Gamma}\|_{R^{m n+n}}<\frac{1}{\left\|\boldsymbol{\Gamma}^{-1}\right\|_{R^{m n+n}}}
$$

holds, from which, by the theorem on the inverse operator [4, p.207], it follows that

the matrix $\boldsymbol{\Gamma}$ is invertible, i.e., the boundary value problem (1) is correctly solvable.

Further, the suggested general scheme of the constructive investigation will be applied to the boundary value problem for the $n$th order differential equation with deviating arguments.

\section{A class of functions and operators}

The constructive techniques for the study of equations with deviating arguments described below are based on a specific approximation of original problems within the class of computable functions and operators [2]. In what follows, we assume that the spaces $D S_{p}^{n}[0, T](m)$ and $W S_{p}^{n}[0, T](m)$ are constructed by means of the partition

$$
0=t_{0}<t_{1}<\cdots<t_{m}<t_{m+1}=T,
$$

where $t_{q}, q=1, \ldots, m+1$, are rational numbers. The sets $B_{q}=\left[t_{q-1}, t_{q}\right), q=1, \ldots, m ; B_{m+1}=$ $\left[t_{m}, T\right]$, and the corresponding characteristic functions $\chi_{q}(\cdot)$ are defined with respect to the same partition. $\operatorname{WS}_{p}^{n}[0, T](m)$ denotes the Banach space of functions $y:[0, T] \rightarrow R^{1}$, with $y^{(n)} \in L_{p}^{1}[0, T], y^{(i)} \in D S_{p}^{1}[0, T](m), i=0, \ldots, n-1$, and the representation

$$
\begin{aligned}
& y(t)=\int_{0}^{t} \frac{(t-s)^{(n-1)}}{(n-1) !} y^{(n)}(s) d s+\sum_{i=0}^{n-1} \frac{t^{i}}{i !} y^{(i)}(0) \\
& +\sum_{i=0}^{n-1} \sum_{q=1}^{m} \frac{\left(t-t_{q}\right)^{i}}{i !} \chi_{\left[t_{q}, T\right]}(t) \Delta y^{(i)}\left(t_{q}\right) ; \\
& \Delta y^{(i)}\left(t_{q}\right)=y^{(i)}\left(t_{q}\right)-y^{(i)}\left(t_{q}-0\right), \\
& \Delta^{n} y=\operatorname{col}\left\{y(0), y^{(1)}(0), \ldots, y^{(n-1)}(0), \Delta y\left(t_{1}\right), \Delta y^{(1)}\left(t_{1}\right), \ldots\right. \text {, } \\
& \left.\Delta y^{(n-1)}\left(t_{1}\right), \ldots, \Delta y^{(1)}\left(t_{m}\right), \Delta y^{(2)}\left(t_{m}\right), \ldots, \Delta y^{(n-1)}\left(t_{m}\right)\right\},
\end{aligned}
$$

with the norm

$$
\|y\|_{W S_{p}^{n}[0, T](m)}=\|\dot{y}\|_{L_{p}^{1}[0, T]}+\left\|\Delta^{n} y\right\|_{m n+n} .
$$


$D_{p}^{n}[0, T]$ denotes the Banach space of absolutely continuous functions $x:[0, T] \rightarrow R^{n}$ such that $\dot{x} \in L_{p}^{n}[0, T]$, with the norm

$$
\|x\|_{D_{p}^{n}[0, T]}=\|x(0)\|_{n}+\|\dot{x}\|_{L_{p}^{n}[0, T]} .
$$

Definition 1 A function $y \in D S_{p}^{n}[0, T](m)$ is said to possess the property $\mathcal{C}$ (is computable) if its components as well as the components of the functions $\dot{y}(\cdot)$ and $\int_{0}^{(\cdot)} y(s) d s$ take rational values at rational values of their arguments.

Let $y \in D S_{p}^{n}[0, T](m)$. The property $\mathcal{C}$ is satisfied by functions $y$ of the form

$$
y(t)=\sum_{q=1}^{m} \chi_{q}(t) p_{q}(t), \quad t \in[0, T]
$$

where the components $p_{q}:[0, T] \rightarrow R^{n}, q=1, \ldots, m$ are polynomials with rational coefficients. We denote by $\mathcal{P}_{m}^{n}$ the set of all $y \in D S_{p}^{n}[0, T](m)$ of the form (7).

Definition 2 A function $y \in W S_{p}^{n}[0, T](m)$ is said to possess the property $\mathcal{C}$ (is computable) if this function and the functions $y^{(i)}(\cdot), i=1, \ldots, n$ and $\int_{0}^{(\cdot)} y(s) d s$ take rational values at rational values of their arguments.

Obviously, the functions $y \in W S_{p}^{n}[0, T](m)$ with $y^{(n)} \in \mathcal{P}_{m}^{n}$ possess the property $\mathcal{C}$.

Definition 3 A function $h:[0, T] \rightarrow R^{1}$ is said to be computable over the partition (6) if $h$ possesses the property $\mathcal{C}$ and for every $j=1, \ldots, m$ there exists an integer $q_{j}, 0 \leq q_{j} \leq j$, such that $h(t) \in B_{q_{j}}$ as $t \in B_{j}$.

An example of a function that is computable over the partition (6) is $h:[0, T] \rightarrow R^{1}$ such that

$$
h(t)=\sum_{q=1}^{m+1} \chi_{q}(t) h_{q}, \quad 0 \leq h_{q} \leq t_{q}, t \in[0, T]
$$

where $h_{q}, q=1, \ldots, m+1$, are rational constants.

Definition 4 A function $h:[0, T] \rightarrow R^{1}$ is said to be computable over the partition (6) in the generalized sense if $h$ possesses the property $\mathcal{C}$, and for every $j=1, \ldots, m$, there exists an integer $q_{j}, 0 \leq q_{j} \leq m+1$, such that $h(t) \in B_{q_{j}}$, as $t \in B_{j}$.

An example of a function that is computable over the partition (6) in the generalized sense is $h:[1, T] \rightarrow R^{1}$ such that

$$
h(t)=\sum_{q=1}^{m+1} \chi_{q}(t) h_{q}, \quad t \in[0, T]
$$

where $h_{q} \in[0, T], q=1, \ldots, m+1$, are rational constants. 
Definition 5 A bounded linear operator $\mathcal{L}: W S_{p}^{n}[0, T](m) \rightarrow L_{p}^{n}[0, T]$ is said to possess the property $\mathcal{C}$ (is computable) if it maps $\mathcal{P}_{m}^{n}$ into itself.

An example of an operator that is computable is

$$
\begin{aligned}
& \left(\mathcal{L}^{n} y\right)(t) \equiv y^{(n)}(t)+\sum_{i=0}^{n-1} \sum_{j=1}^{n_{i}} p_{i j}(t) y^{(i)}\left[h_{i j}(t)\right]=f(t), \\
& y^{(i)}(\xi)=0, \quad \xi \notin[0, T], t \in[0, T],
\end{aligned}
$$

if the coefficients $p_{i j}$ are the elements of the set $\mathcal{P}_{m}^{n}$ and the functions $h_{i j}$ are computable over the partition (6) in the generalized sense.

\section{Problem setting}

Consider the linear boundary value problem

$$
\begin{aligned}
& \left(\mathcal{L}^{n} y\right)(t) \equiv y^{(n)}(t)+\sum_{i=0}^{n-1} \sum_{j=1}^{n_{i}} p_{i j}(t) y^{(i)}\left[h_{i j}(t)\right]=f(t), \\
& y^{(i)}(\xi)= \begin{cases}\phi_{i}^{0}(\xi), & \xi<0, \\
\phi_{i}^{T}(\xi), & \xi>T, \quad t \in[0, T],\end{cases} \\
& \ell^{k} y \equiv \int_{0}^{T} \varphi_{k}(s) y^{(n)}(s) d s+\sum_{i=0}^{n-1} \psi_{i 0}^{k} y^{(i)}(0) \\
& +\sum_{i=0}^{n-1} \sum_{q=1}^{m} \psi_{i q}^{k} \Delta y^{(i)}\left(t_{q}\right)=\beta_{k}, \quad k=1, \ldots, m n+n,
\end{aligned}
$$

where $y \in W S_{p}^{n}[0, T](m), 1 \leq p<\infty, p_{i j}$, and $f \in L_{p}^{1}[0, T]$, the $h_{i j}(\cdot)$ are continuous and strictly monotonic functions over every $B_{q}, i=0, \ldots, n-1, j=1, \ldots, n_{i}$,

$$
\varphi_{k} \in \begin{cases}C S^{1}[0, T](m), & p=1, \\ L_{p^{\prime}}^{1}[0, T], & p>1,\end{cases}
$$

$\beta_{k}$ and $\psi_{i q}^{k} \in R^{1}, k=1, \ldots, m n+n, q=0, \ldots, m . C S^{n}[0, T](m)$ denotes the Banach space of functions $x:[0, T] \rightarrow R^{n}$, defined by the equality

$$
\begin{aligned}
& x(t)=\sum_{q=1}^{m+1} \chi_{q}(t) x_{q}(t), \quad t \in[0, T], \\
& x_{q}(\cdot)=\operatorname{col}\left\{x_{q}^{1}(\cdot), \ldots, x_{q}^{n}(\cdot)\right\},
\end{aligned}
$$

where $x_{q}^{i}, q=1, \ldots, m+1, i=1, \ldots, n$, are continuous functions, and

$$
\|x\|_{C S^{n}[0, T](m)}=\max _{1 \leq i \leq n} \sup _{t \in[0, T]}\left|x^{i}(t)\right| ;
$$

$p^{\prime}$ denotes the adjoint index to $p$ :

$$
p^{\prime}= \begin{cases}\frac{p}{p-1}, & \text { if } p>1, \\ \infty, & \text { if } p=1 .\end{cases}
$$


Consider the principal boundary value problem corresponding to (8):

$$
\begin{aligned}
& \left(\mathcal{L}^{n} y\right)(t) \equiv y^{(n)}(t)+\sum_{i=0}^{n-1} \sum_{j=1}^{n_{i}} p_{i j}(t) y^{(i)}\left[h_{i j}(t)\right]=f(t), \\
& y^{(i)}(\xi)=\left\{\begin{array}{ll}
\varphi_{i}^{0}(\xi), & \xi<0, \\
\varphi_{i}^{T}(\xi), & \xi>T,
\end{array} \quad t \in[0, T],\right. \\
& \Delta^{n} y=\alpha, \quad \alpha=\operatorname{col}\left\{\alpha_{1}, \ldots, \alpha_{m n+n}\right\},
\end{aligned}
$$

under the same assumptions on the problem parameters.

The procedure for the constructive study of the problem (8) consists of the following steps:

- approximation of the problem (8) within the class of computable functions and operators,

- study of the principal boundary value problem (9),

- analysis of its solvability.

\section{Approximation of the problem within the class of computable operators}

Fix $q=1, \ldots, m+1$. Approximate $p_{i j}$ and $f$ on the set $B_{q}$ by polynomials ${ }^{a} p_{i j}^{q}$ and ${ }^{a} f_{q}$ with rational coefficients and define the rational error bounds:

$$
{ }^{v} p_{i j}^{q} \geq\left\|p_{i j}-{ }^{a} p_{i j}^{q}\right\|_{L_{p}^{1}\left[t_{q-1}, t_{q}\right]}, \quad{ }^{v} f_{q} \geq\left\|f-{ }^{a} f_{q}\right\|_{L_{p}^{1}\left[t_{q-1}, t_{q}\right]} .
$$

Now define

$$
{ }^{a} p_{i j}(t)=\sum_{q=1}^{m+1} \chi_{q}(t)^{a} p_{i j}^{q}(t), \quad{ }^{a} f(t)=\sum_{q=1}^{m+1} \chi_{q}(t)^{a} f_{q}(t), \quad t \in[0, T]
$$

for $i=0, \ldots, n-1$ and $j=1, \ldots, n_{i}$.

\section{Approximation of the $h_{i j}$}

Find rational approximations ${ }^{a} \bar{h}_{i j}^{q}$ of $h_{i j}\left(t_{q}\right), i=0, \ldots, n-1, j=1, \ldots, n_{i}, q=1, \ldots, m+1$, and define rational ${ }^{v} h$ such that

$$
{ }^{a} \bar{h}_{i j}^{q}-{ }^{v} h \leq h_{i j}\left(t_{q}\right) \leq{ }^{a} \bar{h}_{i j}^{q}+{ }^{v} h,
$$

and define a rational constant $h_{\triangle}=G C D\left\{{ }^{a} \bar{h}_{i j}^{q}\right\}$. Fix $i=0, \ldots, n-1, j=1, \ldots, n_{i}$, and construct the collection of points $\left\{{ }^{a} h_{i j}^{v}\right\}$ by the rule:

$$
\begin{aligned}
{ }^{a} h_{i j}^{v} & =\min _{0 \leq q \leq m+1}\left\{{ }^{a} \bar{h}_{i j}^{q}\right\}+v \frac{\max _{0 \leq q \leq m+1}\left\{{ }^{a} \bar{h}_{i j}^{q}\right\}-\min _{0 \leq q \leq m+1}\left\{{ }^{a} \bar{h}_{i j}^{q}\right\}}{m_{i j}}, \\
v & =0, \ldots, m_{i j}, m_{i j}=h_{\triangle} \bar{v}_{i j} ;
\end{aligned}
$$

here the positive integer parameter $\bar{v}_{i j}$ defines the accuracy of the approximation to $h_{i j}$, assuming that ${ }^{a} h_{i j}^{v-1}+{ }^{v} h<{ }^{a} h_{i j}^{v}-{ }^{v} h, v=1, \ldots, m_{i j}$. We denote by $v_{i j}^{q}, q=0, \ldots, m+1$, a value 
of $v$ such that ${ }^{a} h_{i j}^{v_{i j}^{q}}={ }^{a} \bar{h}_{i j}^{q}$. For $q=1, \ldots, m+1$, we denote $m_{i j}^{q}+1$ for the number of values ${ }^{a} h_{i j}^{v}$ that belong reliably to the inverse image of $h_{i j}$ in the set $B_{q}$. We define the elements of the set $\mathcal{I}_{i j}^{q}=\left\{{ }^{a} \tilde{h}_{i j}^{q v}\right\}_{\nu=0}^{m_{i j}^{q}}$ as follows:

1. $\quad{ }^{a} \tilde{h}_{i j}^{q v}={ }^{a} h_{i j}^{v_{i j}^{q-1}+v}, v=0, \ldots, m_{i j}^{q}$, if $h_{i j}$ is strictly increasing on $B_{q}$;

2. $a \tilde{h}_{i j}^{q v}={ }^{a} h_{i j}^{v_{i j}^{q}-v}, v=0, \ldots, m_{i j}^{q}$, if $h_{i j}$ is strictly decreasing on $B_{q}$.

Next define the pairs of constants $\left[{ }_{1}^{a} t_{i j}^{q v},{ }_{1}^{v}{ }_{i j}^{q v}\right],\left[{ }_{2}^{a} t_{i j}^{q v},{ }_{2}^{v} t_{i j}^{q v}\right], v=0, \ldots, m_{i j}^{q}$, such that

$$
\begin{aligned}
& h_{i j}^{-1}\left(\tilde{h}_{i j}^{q \nu}\right) \in\left[{ }_{1}^{a} t_{i j}^{q \nu},{ }_{1}^{a} t_{i j}^{q v}+{ }_{1}^{v} t_{i j}^{q \nu}\right] ; \\
& h_{i j}^{-1}\left({ }^{a} \tilde{h}_{i j}^{q \nu}+{ }^{v} h\right) \in\left[{ }_{2}^{a} t_{i j}^{q \nu},{ }_{2}^{a} t_{i j}^{q v}+{ }_{2}^{v} t_{i j}^{q \nu}\right] .
\end{aligned}
$$

We also assume that the following conditions hold:

$$
{ }_{1}^{a} t_{i j}^{q \nu}+{ }_{1}^{v} t_{i j}^{q \nu}<{ }_{2}^{a} t_{i j}^{q \nu} ; \quad t_{q-1}<{ }_{1}^{a} t_{i j}^{q 1} ; \quad{ }_{2}^{a} t_{i j}^{q\left(m_{i j}^{q}-1\right)}+{ }_{2}^{v} t_{i j}^{q\left(m_{i j}^{q}-1\right)}<T,
$$

which can always be satisfied by means of the accuracy of the calculation of the function $h_{i j}^{-1}$ for the given points. Further, we construct the set $\mathcal{J}_{i j}^{q}=\left\{t_{i j}^{q \nu}\right\}, v=0, \ldots, m_{i j}^{q}$,

$$
t_{i j}^{q v}=\frac{{ }_{1}^{a} t_{i j}^{q v}+{ }_{1}^{v} t_{i j}^{q v}+{ }_{2}^{a} t_{i j}^{q v}}{2} .
$$

From the order of the points $t_{i j}^{q \nu}, v=0, \ldots, m_{i j}^{q}$, it follows that

$$
\begin{aligned}
& t_{q-1}=t_{i j}^{q 0}<t_{i j}^{q 1}<\cdots<t_{i j}^{q\left(m_{i j}^{q}-1\right)}<t_{i j}^{q m_{i j}^{q}}=t_{q} ; \\
& { }^{a} \tilde{h}_{i j}^{q \nu}-{ }^{v} h \leq h\left(t_{i j}^{q \nu}\right) \leq{ }^{a} \tilde{h}_{i j}^{q \nu}+{ }^{v} h, \quad v=0, \ldots, m_{i j}^{q} .
\end{aligned}
$$

Next put

$$
\begin{aligned}
& B_{i j}^{q v}=\left[t_{i j}^{q(v-1)}, t_{i j}^{q \nu}\right), \quad v=1, \ldots, m_{i j}^{q}-1 ; \\
& B_{i j}^{q m_{i j}^{q}}= \begin{cases}{\left[t_{i j}^{q\left(m_{i j}^{q}-1\right)}, t_{q}\right),} & q<m+1, \\
{\left[t_{i j}^{q\left(q_{i j}^{k}-1\right)}, T\right],} & q=m+1,\end{cases}
\end{aligned}
$$

and denote by $\chi_{i j}^{q v}(\cdot)$ the characteristic function of $B_{i j}^{q v}, v=1, \ldots, m_{i j}^{q}$. We construct the function ${ }^{a} h_{i j}(\cdot)$ by the following rule:

$$
{ }^{a} h_{i j}(t)=\sum_{q=1}^{m+1} \sum_{\nu=1}^{m_{i j}^{q}} \chi_{i j}^{q \nu}(t)^{a} \tilde{h}_{i j}^{q(v-1)}, \quad t \in[0, T] .
$$

Define the set of points $\mathcal{J}$ by

$$
\mathcal{J}=\bigcup_{i=0}^{n-1} \bigcup_{j=1}^{n_{i}} \bigcup_{q=1}^{m+1} \mathcal{J}_{i j}^{q}
$$


(excluding duplicate elements). By construction, the ${ }^{a} h_{i j}, i=0, \ldots, n-1, j=1, \ldots, n_{i}$, are computable over the partition (12) in the generalized sense.

Below suppose that $\phi_{i}^{0} \in D_{p}^{1}\left[h_{0}^{*}, 0\right], \phi_{i}^{T} \in D_{p}^{1}\left[T, h_{T}^{*}\right], i=0, \ldots, n-1$, and define the constants $h_{0}^{*}$ and $h_{T}^{*}$ by the equalities

$$
\begin{aligned}
& h_{0}^{*}=\min _{\substack{0 \leq i \leq n-1 \\
1 \leq j \leq n_{i}}}\left\{b_{i j}^{0}\right\}, \\
& b_{i j}^{0}=\min \left\{\min _{t \in[0, T]}\left\{h_{i j}(t)\right\}, \min _{0 \leq \nu \leq m_{i j}}\left\{{ }^{a} h_{i j}^{v}-{ }^{v} h\right\}\right\}, \\
& h_{T}^{*}=\max _{\substack{0 \leq i \leq n-1 \\
1 \leq j \leq n_{i}}}\left\{b_{i j}^{T}\right\}, \\
& b_{i j}^{T}=\max \left\{\min _{t \in[0, T]}\left\{h_{i j}(t)\right\}, \max _{0 \leq v \leq m_{i j}}\left\{{ }^{a} h_{i j}^{v}+{ }^{v} h\right\}\right\} .
\end{aligned}
$$

We approximate the functions $\phi_{i}^{0}, \phi_{i}^{T}$ by polynomials ${ }^{a} \phi_{i}^{0},{ }^{a} \phi_{i}^{T}$ with rational coefficients and with rational error bounds $\left\{\begin{array}{l}v \\ 0\end{array} \phi_{i}^{0},{ }_{1}^{v} \phi_{i}^{0}\right\},\left\{{ }_{0}^{v} \phi_{i}^{T},{ }_{1}^{v} \phi_{i}^{T}\right\}$ :

$$
\begin{array}{ll}
{ }_{0}^{v} \phi_{i}^{0} \geq\left|\phi_{i}^{0}\left(h_{0}^{*}\right)-{ }^{a} \phi_{i}^{0}\left(h_{0}^{*}\right)\right|, & { }_{1}^{v} \phi_{i}^{0} \geq\left\|\dot{\phi}_{i}^{0}-{ }^{a} \dot{\phi}_{i}^{0}\right\|_{L_{p}^{1}\left[h_{0}^{*}, 0\right]}, \\
{ }_{0}^{v} \phi_{i}^{T} \geq\left|\phi_{i}^{T}(T)-{ }^{a} \phi_{i}^{T}(T)\right|, & { }_{1}^{v} \phi_{i}^{T} \geq\left\|\dot{\phi}_{i}^{T}-{ }^{a} \dot{\phi}_{i}^{T}\right\|_{L_{p}^{1}\left[T, h_{T}^{*}\right]} .
\end{array}
$$

\section{Approximation of the functional $\ell^{k}$}

The real numbers $\beta_{i}, i=1, \ldots, m n+n$, are approximated by rational numbers ${ }^{a} \beta_{i}$ with rational error bounds ${ }^{v} \beta_{i} \geq\left|\beta_{i}-{ }^{a} \beta_{i}\right|$. The constants $\psi_{i q}^{k}$ are approximated by rational numbers ${ }^{a} \psi_{i q}^{k}$ with rational error bounds ${ }^{v} \psi_{i q}^{k} \geq\left|\psi_{i q}^{k}-{ }^{a} \psi_{i q}^{k}\right|, i=0, \ldots, n-1 ; q=0, \ldots, m$; $k=1, \ldots, m n+n$. On each $B_{q}, q=1, \ldots, m+1$, the functions $\phi_{j}, j=1, \ldots, m n+n$, are approximated by the polynomials ${ }^{a} \phi_{j}^{q}$ with rational coefficients and with rational error bounds ${ }^{v} \phi_{j}^{q} \geq\left\|{ }^{a} \phi_{j}^{q}-\phi_{j}^{q}\right\|_{L_{p^{\prime}}^{1}\left[t_{q-1}, t_{q}\right]}$. Define the functions ${ }^{a} \phi_{j}(\cdot)$ by the equalities

$$
{ }^{a} \phi_{j}(t)=\sum_{q=1}^{m} \chi_{q}(t)^{a} \phi_{j}^{q}(t), \quad t \in[0, T], j=1, \ldots, m n+n .
$$

Let us write the boundary value problem approximating the problem (8) as follows:

$$
\begin{aligned}
\left({ }^{a} \mathcal{L}^{n} y\right)(t) \equiv y^{(n)}(t)+\sum_{i=0}^{n-1} \sum_{j=1}^{n_{i}}{ }^{a} p_{i j}(t) y^{(i)}\left[{ }^{a} h_{i j}(t)\right]={ }^{a} f(t), & \\
y^{(i)}(\xi) & =\left\{\begin{array}{ll}
{ }^{a} \phi_{i}^{0}(\xi), & \xi<0, \\
{ }^{a} \phi_{i}^{T}(\xi), & \xi>T,
\end{array} \quad t \in[0, T],\right. \\
{ }^{a} \ell^{k} y \equiv & \int_{0}^{T}{ }^{a} \varphi_{k}(s) y^{(n)}(s) d s+\sum_{i=0}^{n-1}{ }^{a} \psi_{i 0}^{k} y^{(i)}(0) \\
& +\sum_{i=0}^{n-1} \sum_{q=1}^{m}{ }^{a} \psi_{i q}^{k} \Delta y^{(i)}\left(t_{q}\right)={ }^{a} \beta_{k}, \quad k=1, \ldots, m n+n .
\end{aligned}
$$

Note that the operators ${ }^{a} \mathcal{L}^{n}$ and ${ }^{a} \ell^{k}$ are computable by construction. 


\section{Study of the principal boundary value problem}

The aim of this study is to check whether the problem (9) is correctly solvable, having in mind the computer-assisted proof techniques. This issue is described in detail in [5]. Below suppose that the problem (9) is correctly solvable. Then there exists a fundamental system $y_{k}, k=1, \ldots, m n+n$ of the homogeneous equation

$$
\begin{aligned}
& \left(\mathcal{L}^{n} y\right)(t) \equiv y^{(n)}(t)+\sum_{i=0}^{n-1} \sum_{j=1}^{n_{i}} p_{i j}(t) y^{(i)}\left[h_{i j}(t)\right]=0, \\
& y^{(i)}(\xi)=0, \quad \xi \notin[0, T] ; t \in[0, T],
\end{aligned}
$$

and a fundamental system $\tilde{y}_{k}, k=1, \ldots, m n+n$, of the homogeneous equation

$$
\begin{aligned}
& \left({ }^{a} \mathcal{L}^{n} y\right)(t) \equiv y^{(n)}(t)+\sum_{i=0}^{n-1} \sum_{j=1}^{n_{i}}{ }^{a} p_{i j}(t) y^{(i)}\left[{ }^{a} h_{i j}(t)\right]=0, \\
& y^{(i)}(\xi)=0, \quad \xi \notin[0, T] ; t \in[0, T] .
\end{aligned}
$$

Every function $y_{k}$ is defined as a solution of the principal boundary value problem

$$
\begin{aligned}
& y^{(n)}(t)+\sum_{i=0}^{n-1} \sum_{j=1}^{n_{i}} p_{i j}(t) y^{(i)}\left[h_{i j}(t)\right]=0, \\
& y^{(i)}(\xi)=0, \quad \xi \notin[0, T] ; t \in[0, T], \\
& \Delta^{n} y=\delta_{k}, \quad \delta_{k}=\left\{\delta_{k q}\right\}_{q=1}^{m n+n}, \delta_{k q}= \begin{cases}1, & k=q, \\
0, & k \neq q,\end{cases}
\end{aligned}
$$

and every function $\tilde{y}_{k}$ is defined as a solution of the principal boundary value problem

$$
\begin{aligned}
& y^{(n)}(t)+\sum_{i=0}^{n-1} \sum_{j=1}^{n_{i}}{ }^{a} p_{i j}(t) y^{(i)}\left[{ }^{a} h_{i j}(t)\right]=0, \\
& y^{(i)}(\xi)=0, \quad \xi \notin[0, T] ; t \in[0, T], \\
& \Delta^{n} y=\delta_{k}, \quad \delta_{k}=\left\{\delta_{k q}\right\}_{q=1}^{m n+n}, \delta_{k q}= \begin{cases}1, & k=q, \\
0, & k \neq q,\end{cases}
\end{aligned}
$$

$k=1, \ldots, m n+n$. Denote by ${ }^{a} y_{k}$ an approximation to the functions $\tilde{y}_{k}$ and by ${ }^{v} y_{k}$ the approximation error bounds:

$$
\begin{aligned}
& { }^{a} y_{k}(t)=\sum_{q=1}^{m+1} \chi_{q}(t)_{q}^{a} y_{k}(t), \quad{ }^{v} y_{k}(t)=\sum_{q=1}^{m+1} \chi_{q}(t)_{q}^{v} y_{k}, \quad t \in[0, T], \\
& { }_{q}^{v} y_{k} \geq\left\|\tilde{y}_{k}^{(n)}-{ }_{q}^{a} y_{k}^{(n)}\right\|_{L_{p}^{1}\left[t_{q-1}, t_{q}\right]}, \quad k=1, \ldots, m n+n .
\end{aligned}
$$

A detailed description of the construction of the functions ${ }^{a} y_{k}$ and the estimations of ${ }^{v} y_{k}$, $k=1, \ldots, m n+n$, is given in [5]. 


\section{Analysis of solvability}

Denote the elements of the matrices $\boldsymbol{\Gamma}=\left\{\gamma_{i j}\right\}_{i, j=1}^{m n+n},{ }^{a} \boldsymbol{\Gamma}=\left\{{ }^{a} \gamma_{k j}\right\}_{k, j=1}^{m n+n}$ and ${ }^{v} \boldsymbol{\Gamma}=\left\{{ }^{v} \gamma_{k j}\right\}_{k, j=1}^{m n+n}$ as follows:

$$
\begin{aligned}
& \gamma_{k j} \stackrel{\text { def }}{=} \ell^{k} y_{j}=\int_{0}^{T} \phi_{k}(s) y_{j}^{(n)}(s) d s+\psi_{i_{j} \tau_{j}}^{k} ; \\
& { }^{a} \gamma_{k j} \stackrel{\text { def }}{=} \int_{0}^{T}{ }^{a} \phi_{k}(s)^{a} y_{j}^{(n)}(s) d s+{ }^{a} \psi_{i_{j} \tau_{j}}^{k} ; \\
& { }^{v} \gamma_{k j} \stackrel{\text { def }}{\geq}\left|{ }^{v} \psi_{i_{j} \tau_{j}}\right|+\sum_{q=1}^{m}\left\{\left\|{ }^{a} \psi_{k}^{q}\right\|_{L_{p^{\prime}}^{1}\left[t_{q-1}, t_{q}\right] q}^{v} y_{j}+{ }^{v} \phi_{k}^{q} \times{ }_{q}^{v} y_{j}+{ }^{v} \phi_{k}^{q}\|\|_{q}^{a} y_{j}^{(n)} \|_{L_{p}^{1}\left[t_{q-1}, t_{q}\right]}\right\} ; \\
& i_{j}= \begin{cases}j, & 1 \leq j \leq n ; \\
j-n, & n+1 \leq j \leq 2 n ; \\
j-m n, & m n+1 \leq j \leq m n+n ; \\
\tau_{j}= & \begin{cases}0, & 1 \leq j \leq n ; \\
1, & n+1 \leq j \leq 2 n ; \\
\vdots & \\
m, & m n+1 \leq j \leq m n+n .\end{cases} \end{cases}
\end{aligned}
$$

From definition (18) it follows that

$$
{ }^{v} \gamma_{k j} \geq\left|\gamma_{k j}-{ }^{a} \gamma_{k j}\right|, \quad\left\|\boldsymbol{\Gamma}-{ }^{a} \boldsymbol{\Gamma}\right\|_{R^{(m n+n) \times(m n+n)}} \leq\left\|{ }^{v} \boldsymbol{\Gamma}\right\|_{R^{(m n+n) \times(m n+n)}},
$$

where the matrix $\Gamma$ is defined by (4). Thus we arrived at the following.

Theorem 1 Let the matrix ${ }^{a} \boldsymbol{\Gamma}$ defined by (18) be invertible, and let the inequality

$$
\|v\|_{R^{(m n+n) \times(m n+n)}}<\frac{1}{\left\|\boldsymbol{\Gamma}^{-1}\right\|_{R^{(m n+n) \times(m n+n)}}}
$$

hold. Then the boundary value problem (8) is correctly solvable.

Proof Under the condition of the theorem the inequality

$$
\left\|\boldsymbol{\Gamma}-{ }^{a} \boldsymbol{\Gamma}\right\|_{R^{(m n+n) \times(m n+n)}}<\frac{1}{\left\|{ }^{a} \boldsymbol{\Gamma}^{-1}\right\|_{R^{(m n+n) \times(m n+n)}}}
$$

holds. By the theorem on the inverse operator the matrix $\Gamma$ is defined by (4) to be invertible, i.e., the problem (8) is correctly solvable.

\section{Competing interests}

The author declares that he has not competing interests.

\section{Acknowledgements}

The author expresses his sincere thanks to the referees for the careful and noteworthy reading of the manuscript and helpful suggestions that improved the manuscript.

Received: 12 December 2013 Accepted: 21 February 2014 Published: 12 Mar 2014 


\section{References}

1. Azbelev, NV, Maksimov, VP, Rakhmatullina, LF: Introduction to the Theory of Functional Differential Equations: Methods and Applications. Hindawi Publishing Corporation, New York (2007)

2. Rumyantsev, AN: Reliable computing experiment in the study of boundary problems. Perm State University, Perm (1999) (in Russian)

3. Rumyantsev, AN: The reliable computing experiment in the study of boundary value problems for functional differential equations. Funct. Differ. Equ. 26(3-4), 499-519 (2002)

4. Akilov, GP, Kantorovich, LV: Functional Analysis. Nauka, Moscow (1978) (in Russian)

5. Rumyantsev, AN: The constructive study of differential equations with deviated argument. Mem. Differ. Equ. Math Phys. 26, 91-136 (2002)

10.1186/1687-2770-2014-53

Cite this article as: Rumyantsev: On the constructive investigation of a class of linear boundary value problems for $n$th order differential equations with deviating arguments. Boundary Value Problems 2014, 2014:53

Submit your manuscript to a SpringerOpen ${ }^{\circ}$ journal and benefit from:

- Convenient online submission

- Rigorous peer review

- Immediate publication on acceptance

- Open access: articles freely available online

- High visibility within the field

- Retaining the copyright to your article 